\title{
Incremental - Adaptive - Knowledge Based - Learning for Informative Rules Extraction in Classification Analysis of aGvHD
}

\author{
Maurizio Fiasché ${ }^{1,2}$, Anju Verma ${ }^{2}$, Maria Cuzzola $^{2}$, Francesco C. Morabito ${ }^{1}$, \\ and Giuseppe Irrera ${ }^{2}$ \\ ${ }^{1}$ DIMET, University "Mediterranea" of Reggio Calabria, Italy \\ ${ }^{2}$ Transplant Regional Center of Stem Cells and Cellular Therapy, ”A. Neri”, \\ Reggio Calabria, Italy \\ maurizio.fiasche@unirc.it
}

\begin{abstract}
Acute graft-versus-host disease (aGvHD) is a serious systemic complication of allogeneic hematopoietic stem cell transplantation (HSCT) that occurs when alloreactive donor-derived $\mathrm{T}$ cells recognize host-recipient antigens as foreign. The early events leading to GvHD seem to occur very soon, presumably within hours from the graft infusion. Therefore, when the first signs of aGvHD clinically manifest, the disease has been ongoing for several days at the cellular level, and the inflammatory cytokine cascade is fully activated. So, it comes as no surprise that to identify biomarker signatures for approaching this very complex task is a critical issue. In the past, we have already approached it through joint molecular and computational analyses of gene expression data proposing a computational framework for this disease. Notwithstanding this, there aren't in literature quantitative measurements able to identify patterns or rules from these biomarkers or from aGvHD data, thus this is the first work about the issue. In this paper first we have applied different feature selection techniques, combined with different classifiers to detect the aGvHD at onset of clinical signs, then we have focused on the aGvHD scenario and in the knowledge discovery issue of the classification techniques used in the computational framework.
\end{abstract}

Keywords: EFuNN, gene selection, GvHD, machine learning, wrapper.

\section{Introduction}

Recently, there have been major advances in our knowledge of basic immunology. In parallel, although much of information has been obtained from preclinical models and far less from correlations with clinical observations or treatment, our awareness of the complexity of the pathophysiology of aGvHD is significantly increased [1].

At the same time, the interplay with bioinformatics, defined as the branch of information sciences for the analysis, modelling, simulation and knowledge discovery of biological phenomena, such as genetic processes, has stimulated synergistic research in many cross-disciplinary areas. 
Identifying a compact set of informative genes from microarray data (gene expression data) is critical in the construction of an efficient clinical decision support system. The potential applications of microarray technology are numerous and include identifying markers for classification, diagnosis, disease outcome prediction, target identification and therapeutic responsiveness [2]. Microarray analysis might help to identify unique markers (e.g. a set of gene) of clinical importance. Diagnosis and prediction of a biological state/disease is likely to be more accurate by identifying clusters of gene expression profiles (GEPs) performed by macroarray analysis. Based on a genetic profile, it is possible to set a diagnostic test, so a sample can be taken from a patient, the data related to the sample processed, and a profile related to the sample obtained [2]. This profile can be matched against existing gene profiles and based on similarity, it can be confirmed with a certain probability the presence or the risk for a disease. We apply this approach here to detect acute graft-versus-host disease (aGvHD) in allogeneic hematopoietic stem cell transplantation (HSCT), a curative therapy for several malignant and non malignant disorders. Acute GvHD remains the major complication and the principal cause of mortality and morbility following HSCT [3]. At present, the diagnosis of aGvHD is merely based on clinical criteria and may be confirmed by biopsy of one of the 3 target organs (skin, gastrointestinal tract, or liver) [4]. There is no definitive diagnostic blood test for aGvHD. We have already published a review paper about classification and predictive strategies for validating a novel and not invasive method to confirm the diagnosis of aGvHD in HSCT patients at onset of clinical symptoms [1]. In previous works we have employed global and local models [9][18] and obtained good results using personalized modelling for selecting genes and other important informative biomarkers from clinical, biological and genetic data collected [1][5]. In this paper we want to focus onto another aspect of aGvHD scenario analysis not still approached from anyone. Here we want to extract knowledge and rules from modelling of this disease, so we approached this issue with an incremental adaptive modelling with preliminaries but significant results.

The organization of the rest of this paper is as follows: section 2 explains the data analyzed; two feature selection techniques are applied in pre-processing step in section 3; section 4 describes results obtained with our incremental- adaptiveknowledge based- learning method; section 5 discusses the results of the diagnostic method and section 6 gives conclusions inferred with possible future applications.

\section{Experimental Data}

The goal of this study is to design a model to select a compact set of genes that can profile the pattern of objective microarray data.

For this purpose fifty-nine HSCT patients were enrolled in our study between March 2006 and July 2008 in Transplants Regional Center of Stem Cells and Cellular Therapy "A. Neri" Reggio Calabria, Italy, during a Governative Research Program of minister of the Health with the title: "Project of Integrated Program: Allogeneic Hemopoietic Stem Cells Transplantation in Malignant Hemopathy and Solid Neoplasia Therapy - Predictive and prognostic value for graft vs. host disease of chimerism and gene expression”. Because experimental design plays a crucial role in 
a successful biomarker search, the first step in our design was to choose the most informative specimens and achieve adequate matching between positive cases aGvHD (YES) and negative controls aGvHD (NO) to avoid bias. This goal is best achieved through a database containing high-quality samples linked to quality controlled clinical information. Patients with clinical signs of aGvHD (YES) were selected, and in more than $95 \%$ of them aGvHD was confirmed by biopsy including those with grade I. We used 25 samples from aGvHD (YES) patients that were taken at the time of diagnosis and we selected 60 samples from patients that didn't experienced aGvHD (NO). All together YES/NO patient groups comprised a validation set. Total RNA was extracted from whole peripheral blood samples using a RNA easy Mini Kit (Qiagen) according to the manufacturer's instructions. Reverse transcription of the purified RNA was performed using Superscript III Reverse Transcriptase (Invitrogen). A multigene expression assay to test occurrence of aGvHD were carried out with TaqMan ${ }^{\circledR}$ Low Density Array Fluidic (LDA-macroarray card) based on Applied Biosystems 7900HT comparative dd CT method, according to manufacturer's instructions. Expression of each gene was measured in triplicate and then normalized to the reference gene $18 \mathrm{~S}$ mRNA, who was included in macroarray card. About the project of macroarray card, we selected 47 candidate genes from the published literature, genomic databases, pathway analysis. The 47 candidate genes were involved in immune network and inflammation pathogenesis.

\section{Gene Selection Methods}

The advent of microarray technology emphasized the problem to identify which genes are most important for diagnosing different diseases (e.g. cancer diagnosis) and prognosis task. Feature selection is the process of choosing the most appropriate features (variables) when creating a computational model [2][6]. Generally, most developed gene selection methods can be categorized into two groups: filter and wrapper methods. In this paper we consider two general approaches to feature subset selection, more specifically, wrapper and filter approaches, for gene selection.

Wrappers and filters differ in the way the feature subsets are evaluated. Filter approaches remove irrelevant features according to general characteristics of the data, measuring the intrinsic characteristic of genes. Wrapper approaches, by contrast, apply machine learning algorithms to feature subsets and use cross-validation to evaluate the score of feature subsets. In theory, wrappers should provide more accurate classification results than filters [6][7]. Wrappers use classifiers to estimate the usefulness of feature subsets.

The use of "tailor-made" feature subsets should provide better classification accuracy for the corresponding classifiers, since the features are selected according to their contribution to the classification accuracy of the classifiers. The disadvantage of the wrapper approach is its computational requirement when combined with sophisticated algorithms such as support vector machines.

As a filter approach, CFS was proposed by Hall [8][9]. The rationale behind this algorithm is "a good feature subset is one that contains features highly correlated with the class, yet uncorrelated with each other." It has been shown by Hall[8] and by some of the authors that CFS gave comparable results to the wrapper and executes 
many times faster [10]. It will be shown later in this paper that combining CFS with a suitable classifier, provides a good classification accuracy for diagnosis of aGvHD.

\subsection{Gene Selection: CFS}

Feature Selection is a technique used in machine learning of selecting a subset of relevant features to build robust learning models. The assumption here is that not all genes measured by a macroarray method are related to aGvHD classification. Some genes are irrelevant and some are redundant from the machine learning point of view [2], [11]. It is well-known that the inclusion of irrelevant and redundant information may harm performance of some machine learning algorithms. Feature subset selection can be seen as a search through the space of feature subsets. CFS evaluates a subset of features by considering the individual detector ability of each feature along with the degree of redundancy between them.

$$
C F S s=\frac{k \bar{r}_{c f}}{\sqrt{k+k(k-1) \bar{r}_{f f}}}
$$

Where:

- $\mathrm{CFS}_{S}$ is the score of a feature subset $S$ containing $k$ features,

- $\bar{r}_{c f}$ is the average feature to class correlation ( $\mathrm{f} \in \mathrm{S}$ ),

- $\bar{r}_{f f}$ is the average feature to feature correlation.

The distinction between normal filter algorithms and CFS is that while normal filters provide scores for each feature independently, CFS presents a heuristic "merit" of a feature subset and reports the best subset it finds. To select the genes with CFS, we have:

a) Choose a search algorithm;

b) Perform the search, keeping track of the best subset encountered according to $\mathrm{CFS}_{S}$,

c) Output the best subset encountered.

The search algorithm we used was the best-first with forward selection, which starts with the empty set of genes. The search for the best subset is based on the training data only. Once the best subset has been determined, and a classifier has been built from the training data (reduced to the best features found), the performance of that classifier is evaluated on the test data. The 13 genes selected by CFS are reported in Table 1. A leave-one-out cross validation procedure was performed to investigate the robustness of the feature selection procedures. In 29 runs, the subset of 13 genes was selected 28 times ( $96 \%$ ) by CFS. Now it is possible to use a classifier to estimate the usefulness of feature subsets, and to extract rules describing knowledge about pattern disease.

\subsection{Gene Selection: Wrapper Methods}

While CFS assigns a score to subset of features, Wrapper approaches take biases of machine learning algorithms into account when selecting features. The wrapper 
method applies a machine learning algorithm for a feature subset selection and uses cross-validation to compute a score for them. In general, filters are much faster than wrappers. However, as far as the final classification accuracy is concerned, wrappers normally provide better results. The general argument is that the classifier that will be built from the feature subset should provide a better estimate of accuracy than other methods. The main disadvantage of wrapper approaches is that during the feature selection process, the classifier must be repeatedly called to evaluate a subset.

To select the genes using a wrapper method, we have to:

(a) Choose a machine learning algorithm to evaluate the score of a feature subset.

(b) Choose a search algorithm.

(c) Perform the search, keeping track of the best subset encountered.

(d) Output the best subset encountered.

As a machine learning algorithm, here we used three classifier techniques: simple Bayesian classifier naïve Bayes, a SVM, and an Evolving Fuzzy Neural Network (EFUNN) as knowledge discovery connectionist approach [2]. The naïve Bayes classifier assumes that features are independent given the class. Its performance on data sets with redundant features can be improved by removing such features. A forward search strategy is normally used with naïve Bayes as it should immediately detect dependencies when harmful redundant features are added. SVMs use a kernel function to implicitly map data to a high dimensional space. Then, they construct the maximum margin hyperplane by solving an optimization problem on the training data. Sequential minimal optimization (SMO) [10] is used in this paper to train a SVM with a Linear Kernel after several test emplyed. SVMs have been shown to work well for high dimensional microarray data sets [11]. However, due to the high computational cost it is not very practical to use the wrapper method to select genes for SVMs. Also here the search algorithm was the best-first with forward selection, starting with the empty set of genes. We report here the accuracy of classifiers built from the best feature subset found during the search. The search for the best subset is based on the training data only. Once the best subset has been determined, and a classifier has been built from the training data (reduced to the best features found), the performance of that classifier is evaluated on the test data. The 5 Genes selected using the wrapper method are shown in table 1. Most of the genes selected are also similar to those of the 13 genes selected using the CFS method and the only two genes that are different are actually correlated to other genes from the set of 13 genes. A leaveone-out cross validation procedure was performed to investigate the robustness of the method over the training set: in 29 runs, the subset of 7 genes was selected 26 times $(90 \%)$ by the naïve Bayes wrapper and the group of 5 genes, 29 times (100\%) by the SMO. Section 5 has shown the performance of this technique estimated on the testing data.

\section{Connectionist Model Proposed for Knowledge Discovery Using the Selected Gene Diagnostic Markers}

EFuNNs are learning models that can learn in an incrementally adaptive mode any dataset, regardless of the problem (function approximation, time-series prediction, 
classification, etc.) in a supervised, unsupervised, or hybrid learning mode, subject to appropriate parameter values selected and a certain minimum number of examples presented. Some well-established Neural Networks (NNs) and Artificial Intelligence (AI) techniques have difficulties when applied to incrementally adaptive, knowledgebased learning, for example catastrophic forgetting [12], local minima problem, difficulties to extract rules [2][13], are typical problems in multilayer perceptrons (MLP) and in backpropagation learning algorithm, not being able to adapt to new data without retraining on old ones, and too long training when applied to large datasets. The radial basis function RBF neural networks require clustering to be performed first and then the backpropagation algorithm applied. They are not efficient for incrementally adaptive learning unless they are significantly modified. Many neurofuzzy systems, such as ANFIS [14], FuNN [15], and neofuzzy neuron [16] cannot update the learned rules through continuous training on additional data without suffering catastrophic forgetting. Several analysis and experiments [2] shows that the EFuNN evolving procedure leads to a similar local incrementally adaptive error of other techniques e.g. Resource Allocation Network (RAN) and its modifications, but EFuNNs allow for rules to be extracted and inserted at any time of the operation of the system thus providing knowledge about the problem and reflecting changes in its dynamics. In this respect the EFuNN is a flexible, incrementally adaptive, knowledge engineering model. One of the advantages of EFuNN is that rule nodes in EFuNN represent dynamic fuzzy-2 clusters. Despite the advantages of EFuNN, there are some difficulties when using them, the major is that there are several parameters that need to be optimised in an incrementally adaptive way. Such parameters are: error threshold Err; number, shape, and type of the membership functions; type of learning; aggregation threshold and number of iterations before aggregation, etc. A possible way for solving this problem is a genetic algorithm (GA) use, better a cGA more faster [17]. In the next future an interesting issue could be a study about performance of optimization approach for solving this and other disvantages.

\section{Results}

The dataset described in section 2 that consists of two classes, GvHD(Yes) and $\operatorname{GvHD}(\mathrm{No})$ (that is $\sim \mathrm{GvHD}($ Yes)) and a compact input space, has been used, the expression values of 47 genes has been obtained with the applied biosystem (see section 2). The whole dataset has been divided into training and testing dataset for validation of a classifier system. These two sets came from different patients in different period. A suitable subset of samples for biological peculiarities has been chosen as training data set. The training data set had 29 patient samples (13 $\mathrm{aGvHD}($ Yes) and $16 \mathrm{aGvHD}(\mathrm{No})$ ). The test data set consisted of 30 patient samples (13 aGvHD(Yes) and $17 \mathrm{aGvHD(No)).} \mathrm{The} \mathrm{test} \mathrm{set} \mathrm{shows} \mathrm{a} \mathrm{higher} \mathrm{heterogeneity} \mathrm{with}$ regard to tissue and age of patients making any classification more difficult.

The task is: (1) to find a set of genes distinguishing Yes and Not; (2) to construct a classifier based on these data; and (3) to find a gene profile for each classes. After having applied points 1 and 2 from the methodology above, different subset of genes has been selected. Several EFuNNs are evolved through the N-cross-validation technique (leave one-out method) on the 59 data samples (EFuNN parameters as well 
as are given in Table 2). In the case of data being made available continuously over time and fast adaptation on the new data needed to improve the model performance, online modelling techniques would be more appropriate, so that any new labelled data will be added to the EFuNN and the EFuNN will be used to predict the class of any new unlabelled data. This is an aim for future developments.

Different EFuNN were evolved with the use of different sets of genes as input variables. The question of which is the optimum number of genes for a particular task is a difficult one to answer. Table 3 shows an example of the extracted rules after all samples, each of them having only 13 genes filtered by CFS, are learned by the EFuNN. The rules are 'local' and each of them has the meaning of the dominant rule in a particular subcluster of each class from the input space. Each rule covers a cluster of samples that belong to a certain class. These samples are similar to a degree that is defined as the radius of the receptive field of the rule node representing the cluster. For example, Rule 1 from Table 3 shows that 7 samples of class 1 (GvHD YES) are similar in terms of having genes g1, g2 and g9 overexpressed, and at the same time genes g6 and g7 are underexpressed. One class may be represented by several rules, profiles, each of them covering a subgroup of similar samples. This can lead to a new investigation on why the subgroups are formed and why they have different profiles (rules), even being part of the same class (in this case for the four grading of aGvHD). The extracted rules for each class comprise a profile of this class, our next issue will be visualize this pattern in a significant way.

\section{Biomedical Conclusions and Future Work}

We examined the immune transcripts to study the applicability of gene expression profiling (macroarray) as a single assay in early diagnosis of aGvHD. Our interest was to select fewer number of molecular biomarkers from an initial gene panel and exploiting this to develop a fast, easy and non-invasive diagnostic test [1][5] [18], being able to extract rules and knowledge for modelling the disease. The proposed method provides a good overall accuracy to confirm aGvHD development in HSCT setting. From a biological point of view, the results are reliable. Others have reasoned that Th2 cell therapy could rapidly ameliorate severe aGvHD via IL-4 and IL-10 mediated mechanisms [19][20]. It is noteworthy that in our study a set of genes, indicated by computational analysis, included same mediators of Th2 response such as IL10, and signal transducer and activator of transcription 6, interleukin-4 induced (STAT6). All these were strongly down-regulated in aGvHD (YES) setting, suggesting absence of control mediated by Th2 cells. Therefore, we highlight the fact that defective expression of ICOS impaired the immune protective effectors during clinical aGvHD. This evidence is supported by a previous report about ICOS as regulatory molecule for $\mathrm{T}$ cell responses during aGvHD. It has been showed that ICOS signal inhibits aGvHD development mediated by CD8 positive effector cells in HSCT [20]. According to previous reports, mediators of apoptosis cells and dendritic cell activators were involved. In our study increased expression levels of CXCL10 and CCL7 were identify as informative biomarker of alloreactive disease. Altogether our results strongly outlined the importance and utility of non-invasive tool for aGvHD diagnosis based on GEP. We believe that to achieve an advantage from GEP 
performance, it is very important to know: a) the transcript levels of immune effector cells in early time post-engraftment in order to better understand polarization of $\mathrm{Th} 2$ cells; b) the CD8 positive cell action. As a clinical trial, tissue biopsies were performed to confirm the above diagnostic results. In conclusion, our models may prevent the need for an invasive procedure as already discussed in [1][5][9] and it is possible to extract knowledge and rules after features selection task with wrappers and filters combined with a suitable classifier. This study demonstrated, for the first time, that the proposed incremental- adaptive- knowledge based learning procedure used for integrating the framework tool for diagnosis of aGvHD [1][5][18] confirms a satisfactory $97 \%$ accuracy over independent test data set of HSCT population and return rules for individuating gene profiles for this complex disease. We plan to extend the system as a personalized model [18][21] including all clinical and genetic variables, testing with new data samples this method and for a larger group of patients to capture their peculiarity. Moreover a visualization technique for distinguishing different profiles needed and at last novel classifiers can be explored. The authors are engaged in this direction.

Table 1. The 13 genes selected from CFS with their names and meaning, the 7 genes selected through the wrapper- naïve Bayes method are marked with ${ }^{\circ}$, the 5 genes selected with SVM are marked with*

\begin{tabular}{|c|c|c|}
\hline $\begin{array}{l}\text { Gene } \\
\text { Name }\end{array}$ & Official full name & Immune function \\
\hline BCL2A1 & BCL2-related protein A1 & Anti- and pro-apoptotic regulator. \\
\hline CASP $1^{\circ} *$ & $\begin{array}{l}\text { Caspase } 1, \quad \text { apoptosis- } \\
\text { related } \\
\text { peptidase }\end{array}$ & $\begin{array}{l}\text { Central role in the execution-phase of cell } \\
\text { apoptosis. }\end{array}$ \\
\hline CCL7 & $\begin{array}{l}\text { chemokine (C-C motif) } \\
\text { ligand } 7\end{array}$ & Substrate of matrix metalloproteinase 2 \\
\hline CD83 & CD83 molecule & Dendritic cells regulation. \\
\hline $\mathrm{CXCL}^{\circ} 0^{\circ}$ & $\begin{array}{l}\text { chemokine }(\mathrm{C}-\mathrm{X}-\mathrm{C} \\
\text { motif) ligand } 10\end{array}$ & $\begin{array}{l}\text { Pleiotropic effects, including stimulation of } \\
\text { monocytes, natural killer and T-cell migration, } \\
\text { and modulation of adhesion molecule expression. }\end{array}$ \\
\hline $\mathrm{EGR}^{\circ}{ }^{\circ}$ & Early growth response 2 & $\begin{array}{l}\text { transcription factor with three tandem } \mathrm{C} 2 \mathrm{H} 2 \text {-type } \\
\text { zinc fingers. }\end{array}$ \\
\hline FAS & $\begin{array}{l}\text { TNF receptor } \\
\text { superfamily, member 6) }\end{array}$ & $\begin{array}{l}\text { Central role in the physiological regulation of } \\
\text { programmed cell death. }\end{array}$ \\
\hline $\operatorname{ICOS}^{\circ} *$ & $\begin{array}{l}\text { Inducible T-cell co- } \\
\text { stimulator }\end{array}$ & $\begin{array}{l}\text { Plays an important role in cell-cell signaling, } \\
\text { immune responses, and regulation of cell } \\
\text { proliferation. }\end{array}$ \\
\hline IL4 & Interleukin 4 & Immune regulation. \\
\hline $\mathrm{IL}^{\circ} 0^{\circ} *$ & Interleukin 10 & Immune regulation. \\
\hline SELP & selectin P & Correlation with endothelial cells. \\
\hline $\mathrm{SLPI}^{\circ}$ & Stomatin (EPB72)-like 1 & Elemental activities such as catalysis. \\
\hline STAT6 & $\begin{array}{l}\text { transducer and activator } \\
\text { of transcription } 6 \text {, } \\
\text { interleukin- } 4 \text { induced }\end{array}$ & Regulation of IL4- mediated biological responses. \\
\hline Foxp-3 * & forkhead box P3 & $\begin{array}{l}\text { Regulatory } \mathrm{T} \text { cells play important roles in the } \\
\text { maintenance control of transplantation tolerance. }\end{array}$ \\
\hline $\mathrm{CD} 52^{\circ} *$ & CD52 antigen & B-cell activation. \\
\hline
\end{tabular}


Table 2. (a)EFuNN-1: Experimental results of wrapper with EFuNN as classifier and EFuNN2: Experimental results of a CFS with EFuNN classifier. The parameter values and error results of N-cross-validation (leave-one-out method) EFuNN models for dataset described in section 2.(b)Experimental results of a CFS with EFuNN classifier and a wrapper method combined with SVM. The starting set has been divided in training set and test set, a leave one-out crossvalidation has been calculated for the two subsets.

(a)

\begin{tabular}{llllll}
\hline Model & Errthr & Rmax & $\begin{array}{l}\text { Rule } \\
\text { Nodes }\end{array}$ & $\begin{array}{l}\text { Classification Accuracy } \\
\text { - Training data }\end{array}$ & $\begin{array}{l}\text { Classification } \\
\text { Accuracy - Test data }\end{array}$ \\
\hline EFuNN-1 & 0.9 & 0.4 & 6.3 & 97.4 & 97.0 \\
EFuNN-2 & 0.9 & 0.5 & 4.0 & 95.0 & 97.2 \\
\hline
\end{tabular}

(b)

\begin{tabular}{lll}
\hline Method & Training set & Test set \\
\hline CFS-EFuNN & $28(29)$ & $29(30)$ \\
Wrapper-SVM & $29(29)$ & $29(30)$ \\
Wrapper-NaiveB. & $26(29)$ & $29(30)$ \\
Wrapper- EFuNN & $28(29)$ & $29(30)$ \\
\hline
\end{tabular}

Table 3. 7 samples of class 1 (GvHD YES) are similar in terms of having genes g1, g2 and g9 overexpressed, and at the same time genes $\mathrm{g} 6$ and $\mathrm{g} 7$ are underexpressed

\section{Rule 1:}

if [g1] is $(10.8) \&[\mathrm{~g} 2]$ is $(10.96) \&[\mathrm{~g} 6]$ is $(20.7) \&[\mathrm{~g} 7]$ is $(20.9) \&[\mathrm{~g} 9]$ is $(10.89)$ receptive field $=0.1$ (radius of cluster), then class 1 , accomodated training samples $=7 / 30$

Rule 4:

[g3] is $(20.87) \&[\mathrm{~g} 5]$ is $(10.83) \&$ [g6] is $(20.8) \&$ [g7] is $(10.9) \&[\mathrm{~g} 9]$ is $(10.78) \&[\mathrm{~g} 11]$ is $(20.95)$ receptive field $=0.102$ (radius of cluster), then class 2 , accomodated training samples $=9 / 30$
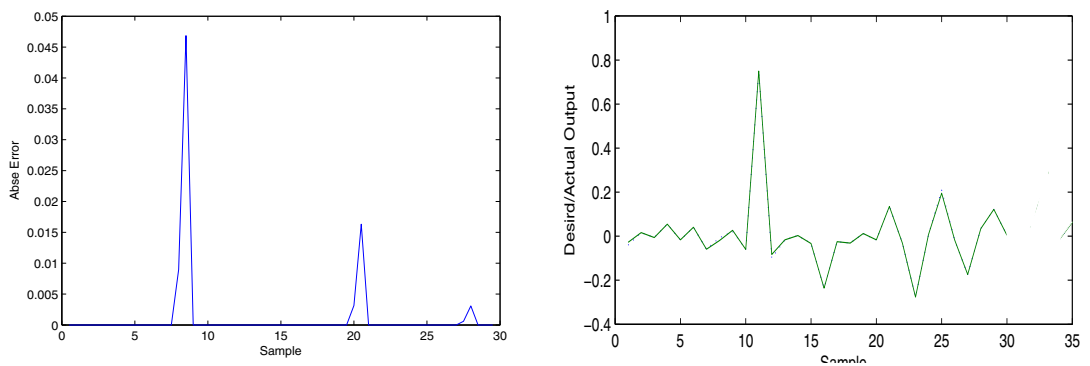

Fig. 1. Absolute error and desidere output plots for aGvHD data after filtering dataset with CFS. EFuNN-1 has been applied for obtaining these results.

Acknowledgments. This study has been supported by Italian Minister of the Health and from Calabria Region. Many thanks to doctors and biologist of CTMO and to Prof. Nik Kasabov for their support during this project. 


\section{References}

1. Fiasché, M., Cuzzola, M., Irrera, G., Iacopino, P., Morabito, F.C.: Advances in Medical Decision Support Systems for Acute Graft-versus-Host Disease: Molecular and Computational Intelligence Joint Approaches. In: Frontiers in Biology. Higher Education Press and Springer -Verlag GmbH, doi:10.1007/s11515-011-1124-8

2. Kasabov, N.: Evolving Connectionist Systems: The Knowledge Engineering Approach, 2nd edn. Springer, London (2007)

3. Weisdorf, D.: Graft vs. Host disease: pathology, prophylaxis and therapy: GvHD overview. Best Pr. \& Res. Cl. Haematology 21(2), 99-100 (2008)

4. Ferrara, J.L.: Advances in the clinical management of GvHD. Best Pr. \& Res. Cl. Haematology 21(4), 677-682 (2008)

5. Fiasché, M., Cuzzola, M., Iacopino, P., Kasabov, N., Morabito, F.C.: Personalized Modeling based Gene Selection for acute GvHD Gene Expression Data Analysis: a Computational Framework Proposed. Australian Journal of Intelligent Information Processing Systems 12(4) (2010), Machine Learning Applications (Part II)

6. Langley, P.: Selection of relevant features in machine learning. In: Proceedings of AAAI Fall Symposium on Relevance, pp. 140-144 (1994)

7. Guyon, I., Elisseeff, A.: An introduction to variable and feature selection. The Journal of Machine Learning Research 3, 1157-1182 (2003)

8. Hall, M.A.: Correlation-based feature selection for machine learning. Ph.D. Thesis. Department of Computer Science, University of Waikato, New Zealand

9. Fiasché, M., Verma, A., Cuzzola, M., Iacopino, P., Kasabov, N., Morabito, F.C.: Discovering Diagnostic Gene Targets for Early Diagnosis of Acute GvHD Using Methods of Computational Intelligence on Gene Expression Data. Journal of Artificial Intelligence and Soft Computing Research 1(1), 81-89 (2011)

10. Platt, J.: Fast training of support vector machines using sequential minimal optimization. In: Advances in Kernel Methods-Support Vector Learning. MIT Press, Cambridge (1998)

11. Furey, T.S., Cristianini, N., Duffy, N., Bednarski, D.W., Schummer, M., Haussler, D.: Support vector machine classification and validation of cancer tissue samples using microarray expression data. Bioinformatics 16, 906-914 (2000)

12. Robins, A.: Consolidation in neural networks and the sleeping brain. Connection Sci. 8(2), 259-275 (1996)

13. Duch, W., Adamczak, R., Grabczewski, K.: Extraction of logical rules from neural networks. Neural Proc. Lett. 7, 211-219 (1998)

14. Jang, R.: ANFIS: Adaptive network based fuzzy inference system. IEEE Trans. Syst. Man Cybern. 23(3), 665-685 (1993)

15. Kasabov, N., Kim, J.S., Watts, M., Gray, A.: FuNN/2 - A fuzzy neural network architecture for adaptive learning and knowledge acquisition. Inf. Sci. Appl. 101(3-4), 155-175 (1997)

16. Yamakawa, T., Uchino, E., Miki, T., Kusanagi, H.: A neo fuzzy neuron and its application to system identification and prediction of the system behaviour. In: Proceedings of the Second International Conference on Fuzzy Logic \& Neural Networks, Iizuka, Japan, pp. 477-483 (1992)

17. Harik, G.R., Lobo, F.G., Goldberg, D.E.: The compact genetic algorithm. IEEE Trans. Evolutionary Computation 3(4), 287-297 (1999) 
18. Fiasché, M., Cuzzola, M., Fedele, R., Iacopino, P., Morabito, F.C.: Machine Learning and Personalized Modeling based Gene Selection for acute GvHD Gene Expression Data Analysis. In: Diamantaras, K., Duch, W., Iliadis, L.S. (eds.) ICANN 2010, Part I. LNCS, vol. 6352, pp. 217-223. Springer, Heidelberg (2010)

19. Foley Jason, J.E., Mariotti, J., Ryan, K., Eckhaus, M., Fowler, D.H.: The cell therapy of established acute graft-versus-host disease requires IL-4 and IL-10 and is abrogated by IL2 or host-type antigen-presenting cells. Biology of Blood and Marrow Transplantation 14, 959-972 (2008)

20. Paczesny, S., Hanauer, D., Sun, Y., Reddy, P.: New perspectives on the biology of acute GvHD. Bone Marrow Transplantation, 45-1-45-11 (2010)

21. Kasabov, N.: Global, local and personalised modelling and profile discovery in Bioinformatics: An integrated approach. Pattern Recognition Letters 28(6), 673-685 (2007) 